\title{
The Glycosylation and in Vivo Stability of Human Granulocyte- Macrophage Colony-Stimulating Factor Produced in Rice Cells
}

\author{
Hyoung Jin KIm, ${ }^{a}$ Dong Hoon LeE, ${ }^{a}$ Dae Kyong KIm,${ }^{a}$ Gyu-Bum Han,${ }^{b}$ and Hong-Jin KIm ${ }^{*}, a$ \\ ${ }^{a}$ College of Pharmacy, Chung-Ang University; 221 Huksuk-Dong, Dongjak-ku, Seoul 156-756, South Korea: and \\ ${ }^{b}$ Hanson Biotech Co., Ltd.; 461-6 Jeonmin-dong, Yuseong-gu, Daejeon 305-811, South Korea. \\ Received October 4, 2007; accepted November 5, 2007; published online November 6, 2007
}

\begin{abstract}
Recombinant human granulocyte-macrophage colony-stimulating factor (rhGM-CSF) has been produced in several different cell types, and has different properties depending on the production process used. There has been no report of glycosylation ratio or of the role of glycans for plant cells-derived rhGM-CSF. We investigated the terminal sialylation of rhGM-CSF produced in rice cells (rrhGM-CSF) using lectins. Glycosylation ratios of rrhGM-CSF and yeast-derived rhGM-CSF (yrhGM-CSF) were evaluated by chemical deglycosylation. After intravenous administration to rats, the clearance rates of rrhGM-CSF and yrhGM-CSF as well as deglycosylated forms of them were evaluated. In vitro bioactivities of rrhGM-CSF and yrhGM-CSF were measured in dose- and time-dependent manners. Although rrhGM-CSF does not have terminal sialic acids, the glycosylation ratio was two-fold higher than that of yrhGM-CSF, and rrhGM-CSF sustained longer than yrhGM-CSF in the blood circulation. Moreover, yrhGM-CSF and rrhGM-CSF have almost same bioactivity via dose- and time-dependent manners. Deglycosylated rrhGM-CSF was cleared faster than native forms, while deglycosylated yrhGM-CSF and yrhGM-CSF were similarly cleared in blood circulation. These results suggest that the glycans on rrhGMCSF are superior to the glycans on yrhGM-CSF in terms of in vivo stability.
\end{abstract}

Key words granulocyte-macrophage colony-stimulating factor; lectin; sialic acid; chemical deglycosylation

Human granulocyte-macrophage colony-stimulating factor (hGM-CSF) is a glycoprotein hemopoietic growth factors that stimulates the proliferation of myeloid precursor cells and activates mature granulocytes and macrophages. ${ }^{1)}$ Recombinant hGM-CSF (rhGM-CSF) is produced by a number of cell types including lymphocytes, endothelial cells, monocytes, fibroblasts, and some malignant cells. ${ }^{2)} \mathrm{cDNA}$ of hGM-CSF has been cloned and used to produce rhGM-CSF in cultured animal cells, ${ }^{3,4)}$ yeast, ${ }^{5)}$ Escherichia coli ${ }^{6,7)}$ and plant cells. ${ }^{8-11)}$ hGM-CSF has two potential $N$-glycosylation sites (Asn 27, Asn 37) and three potential $O$-glycosylation sites (Ser 7, Ser 9, Thr 10), ${ }^{4,12,13)}$ Escherichia coli-derived rhGM-CSF has a molecular weight of $14.5 \mathrm{kDa}$ and is nonglycosylated. ${ }^{7)}$ Yeast-derived rhGM-CSF has a molecular weight of $16-18 \mathrm{kDa}$ and is $O$-glycosylated, which contains leucine instead of proline at position 23 of mature form of wild-type hGM-CSF. ${ }^{14,15)}$ Human lymphoblastoid Namalwa cell-derived rhGM-CSF molecules are $O$-glycosylated and produced in $0 \mathrm{~N}, 1 \mathrm{~N}$ and $2 \mathrm{~N}$ forms, according to the degree of $N$-glycosylation. ${ }^{14)} \mathrm{N}$-Glycosylation of hGM-CSF has an important role in prolonging its half-life in the blood stream. ${ }^{13,14)}$ Therefore, the in vivo stability of heavily glycosylated animal cell-derived rhGM-CSF is superior to that of the yeast and Escherichia coli-derived forms. ${ }^{14)}$ Transgenic plant-derived rhGM-CSF has been produced in tobacco cells, ${ }^{8)}$ tomato cells, ${ }^{10)}$ sugarcane ${ }^{11)}$ and rice cells. ${ }^{9)}$ The most important features of production in transgenic plants are its very low investment and operating costs. ${ }^{16,17)}$ Downstream processing has obvious economic advantages over traditional fermentation methods because recombinant proteins can be produced in plants at $2-10 \%$ of the cost of microbial fermentation systems and at $0.1 \%$ of the cost of mammalian cell culture. ${ }^{18,19)} \mathrm{N}$-Linked glycans in plant have a core substituted by two $\mathrm{N}$-acetylglucosamine (GlcNAc) residues, as observed in mammals. However the plant $\mathrm{N}$-glycans lacks ter- minal sialic acids, and have plant-specific $\alpha 1,3$-fucose and $\beta 1,6$-xylose residues. ${ }^{20)}$ The absence of terminal sialic acids in glycoproteins markedly decreases their in vivo stability. ${ }^{21,22)}$ However, recent reports of Shah et al. ${ }^{23)}$ and Takashima et al. ${ }^{24)}$ suggest a possible genetic and enzymatic basis for sialylation in plants and thus the potential for terminal sialylation of glycoproteins produced in plant cells. However there has been no report of terminal sialylation or of the role of glycans in glycoproteins produced in plant cells.

In this study, we analyzed the terminal sialylation of rice cell-derived rhGM-CSF (rrhGM-CSF). We measured the in vitro bioactivities of rrhGM-CSF and commercial yeast-derived rhGM-CSF (yrhGM-CSF) and evaluated the dependence of their in vivo stabilities on glycosylation by chemically deglycosylating them.

\section{MATERIALS AND METHODS}

Proteins For obtaining the rrhGM-CSF, mRNA of hGMCSF was isolated from peripheral blood mononuclear cells and cloned as described in Shin et al. ${ }^{9)}$ The hGM-CSF expression vector pMYN44 containing the hGM-CSF gene under the control of the Ramy3D promoter and with a signal peptide coding sequence was transfected into rice cells and transgenic derivatives were selected with hygromycin B. mRNA and proteins were obtained from suspension cell cultures and were identified by Northern and Western blotting, respectively. ${ }^{9)}$ Proteins were purified by DEAE anion-exchange chromatography. Leucogen (Sargramostim, LG Life Science, Seoul, Korea) was used for yrhGM-CSF. The yrhGM-CSF was used for chemical deglycosylation, pharmacokinetic experiment and in vitro bioactivity assay, as a control.

Preparation of Asialo Chinese Hamster Ovary Cell-Derived Recombinant Human Erythropoietin (CHO-EPO) 
Terminal sialic acids were removed with $\alpha(2 \rightarrow 3,6,8,9)$ neuraminidase, proteomics grade, from Arthrobacter ureafaciens with $5 \times$ reaction buffer (Sigma, St. Louis, MO, U.S.A.). $170 \mu \mathrm{g}$ of samples and neuraminidase mixture were incubated at $37^{\circ} \mathrm{C}$ for $3 \mathrm{~h}$ and the reactions were stopped by heating for $5 \mathrm{~min}$ at $100^{\circ} \mathrm{C} .^{25,26)}$ The solutions were dialyzed against distilled water to remove remaining sialic acids. $\mathrm{Re}$ moval of terminal sialic acids was confirmed by examining terminal sialylation using lectins, as described below.

Analysis of Terminal Sialylation $\alpha 2,3$ and $\alpha 2,6$-Terminal sialylation of rrhGM-CSF was determined using biotinylated Maackia amurensis lectin II (MAA II) (Vector Laboratories, Burlingame, CA, U.S.A.) and biotinylated Sambucus nigra lectin (SNA) (Vector Laboratories), respectively. ${ }^{27)}$ 96well microtitre plates were coated overnight at $4{ }^{\circ} \mathrm{C}$ with $30 \mathrm{ng}$ of sample per well. Unbound proteins were removed by washing three times with Tris buffered saline (TBS) containing $0.1 \%$ Tween 20 (TBST) and the plates were incubated with TBST for $1 \mathrm{~h}$ at $37^{\circ} \mathrm{C}$. After washing twice with $1 \mathrm{~mm}$ Tris pH 7.5 containing $0.1 \%$ Tween 20 and $1 \mathrm{~mm} \mathrm{CaCl}_{2}$, $1 \mathrm{~mm} \mathrm{MgCl}, 1 \mathrm{~mm} \mathrm{MnCl}_{2}$ and biotin-labeled lectins were added and left at $37^{\circ} \mathrm{C}$ for $1 \mathrm{~h}$. The plates were washed four times with TBST, streptavidin-HRP (PIERCE, Rockford, IL, U.S.A.) was added and incubation continued at room temperature for $30 \mathrm{~min}^{27)}$ Color reactions were developed using TMB substrate and measured at $450 \mathrm{~nm}$. Values are shown as averages and standard deviations of duplicate measurements.

Chemical Deglycosylation Deglycosylation of rrhGMCSF and yrhGM-CSF was performed with trifluoromethanesulfonic acid (TFMS, Sigma). Pre-cooled 10\% anisole in TFMS solution was added to $1 \mathrm{mg}$ of lyophilized rhGM-CSF sample and the mixture was intermittently shaken for $3 \mathrm{~h}$ at $4{ }^{\circ} \mathrm{C}$. $0.2 \%$ bromophenol blue solution (indicator) was added followed by pre-cooled $60 \%$ pyridine solution until the color changed to light purple or blue. ${ }^{28-30)}$ The solution was dialyzed against distilled water to remove remaining glycans and the deglycosylated rhGM-CSF was determined by SDSPAGE analysis. Concentrations were measured by sandwich ELISA using an OptEIA ${ }^{\mathrm{TM}}$ set (BD Bioscience, San Jose, CA, U.S.A.).

SDS-PAGE All samples were subjected to 12\% PAGE in the presence of sodium dodecyl sulfate according to the method of Laemmli. ${ }^{31)}$ Separated proteins were visualized by silver staining (Amersham Pharmacia Biotech, Uppsala, Sweden).

Clearance Experiment in the Rat Three independent pharmacokinetics experiments were performed, using six weeks old male Sprague-Dawley rats (155-165 g) (Orientbio, Kyonggi, Korea). Animals were acclimated for $7 \mathrm{~d}$ under controlled environmental conditions with free access to solid food and water. The animals were maintained at a constant temperature of $21 \pm 1^{\circ} \mathrm{C}, 12 \mathrm{~h}$ light/dark cycle. The protocol of this study was approved by the Animal Care and Use Committee of the College of Pharmacy, Chung-Ang University. Groups of three male rats received single intravenous injections of rrhGM-CSF, yrhGM-CSF, deglycosylated rrhGMCSF or deglycosylated yrhGM-CSF, each at a dose of 180 $187 \mu \mathrm{g} / \mathrm{kg}$. Blood samples were withdrawn at $0.25,1,2,4,8$, 10,24 and $48 \mathrm{~h}$ post injection. ${ }^{32)}$ These were centrifugated and the sera were stored at $-80{ }^{\circ} \mathrm{C} .{ }^{14,32)}$ The serum protein levels of rhGM-CSFs were determined by sandwich ELISA using an OptEIA ${ }^{\mathrm{TM}}$ set (BD Bioscience).

In Vitro Bioactivity Assay Bioactivities of rrhGM-CSF and yrhGM-CSF were determined using the human erythroleukemic cell line (TF-1 cell line). The TF-1 cells were maintained in RPMI-1640 (GIBCO BRL, Grand Island, NY, U.S.A.) containing 10\% FBS. To investigate dose-dependent effects of GM-CSFs, aliquots $\left(100 \mu \mathrm{l} ; 5 \times 10^{4}\right.$ cells $)$ of a cell suspension were incubated in wells of flat-bottomed 96-well tissue culture plate for $24 \mathrm{~h}$. rhGM-CSF samples were serially diluted to $100-10000 \mathrm{pg} / \mathrm{ml}$ with medium and added to the plates. To investigate time-dependent effects of rhGMCSFs, aliquots $\left(1 \mathrm{ml} ; 1 \times 10^{5}\right.$ cells $)$ of a cell suspension were incubated in wells of flat-bottomed 6-well tissue culture plate for $24 \mathrm{~h} .10000 \mathrm{pg} / \mathrm{ml}$ of rhGM-CSF samples added to the plates. After shaking for $1 \mathrm{~min}, 1 \mathrm{Ci} / \mathrm{ml}$ of $[3 \mathrm{H}]$ thymidine was added and the plates were incubated for $24 \mathrm{~h}$ and $48 \mathrm{~h}$ at $37^{\circ} \mathrm{C}$ in a humidified atmosphere of $5 \% \mathrm{CO}_{2}$. The cells were washed three times and lysed with $10 \%$ Triton X-100. The lysates were centrifuged for $10 \mathrm{~min}$ at $10000 \mathrm{~g}$, and the radioactivity of the supernatants was measured with a $\beta$ counter. ${ }^{33)}$

\section{RESULTS}

Terminal Sialylation of rrhGM-CSF $\alpha 2,3$-Terminal sialic acids of rrhGM-CSF were detected using MAA II (Fig. 1A). The positive control, CHO-EPO has only $\alpha 2,3$-linked sialic acids due to the absence of a $\alpha 2,6$-sialyltransferase in Chinese hamster ovary (CHO) cells. ${ }^{34,35)}$ As shown in Fig. $1 \mathrm{~A}$, the optical density of CHO-EPO was over 0.8 , whereas that of non-glycosylated BSA (negative control) was less
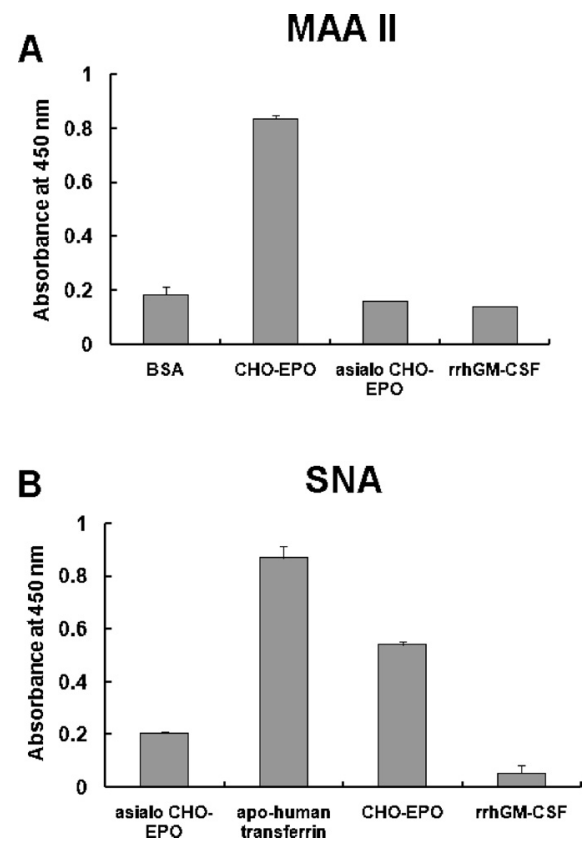

Fig. 1. Analysis of $\alpha 2,3-$ and $\alpha 2,6$-Terminal Sialylation

$\alpha 2,3$-terminal sialic acids of rrhGM-CSF were detected using biotinylated Maackia amurensis lectin II (MAA II) (A) and $\alpha 2,6$-terminal sialic acids of rrhGM-CSF were detected using biotinlyated Sambucus nigra Lectin (SNA) (B). MAA II and SNA were detected using poly-HRP streptavidin. The values were measured at OD $450 \mathrm{~nm} . \alpha 2,3-$ terminal sialylation of rrhGM-CSF was compared with BSA (negative control) and EPO produced in CHO cells (CHO-EPO, positive control). $\alpha 2$,6-terminal sialylation of rrhGM-CSF was compared with apo-human transferrin (positive control) and asialo CHO-EPO (negative control). Values are means \pm S.D. of duplicate measurements. Asialo refers to the desialylated form. 
than 0.2. Asialo CHO-EPO (terminal sialic acids removed by neuraminidase) had the same optical density as BSA, confirming removal of $\alpha 2,3$-linked sialic acids (Fig. 1A). rrhGM-CSF had the same optical density as BSA and asialo CHO-EPO. Therefore, $\alpha 2,3$-linked sialic acid was not detected in rrhGM-CSF (Fig. 1A).

The $\alpha 2,6$-terminal sialic acids of rrhGM-CSF were detected using SNA (Fig. 1B). SNA primarily binds to $\alpha 2,6-$ linked sialic acids and secondarily to $\alpha 2,3$-linked sialic acids. ${ }^{36}$ The positive control, apo-human transferrin is known to have only $\alpha 2,6$-linked sialic acids. ${ }^{37)}$ As shown Fig. $1 \mathrm{~B}$, apo-human transferrin had the highest value followed by CHO-EPO. This result agrees with the properties of SNA. The value for asialo CHO-EPO was about 0.2 and for rrhGM-CSF less than 0.2 (Fig. 1B). Therefore, $\alpha 2,6$-linked sialic acid was not detected in rrhGM-CSF (Fig. 1B). These results indicate that mammals like $\alpha 2,3-$ and $\alpha 2,6$-linked terminal sialic acid do not exist enough to be detected or are not contained in rrhGM-CSF.

Deglycosylation of rrhGM-CSF and yrhGM-CSF TFMS removes $O$-glycans and $N$-glycans while not affecting other post-translational modifications or amino acids of glycoprotein $^{29,38,39)}$ This property of TFMS is useful for determining the glycosylation ratio of uncharacterized glycoproteins. ${ }^{40-42}$ TFMS is particularly useful for removing plant glycans that are not removed by enzymatic deglycosylation. ${ }^{29)}$ After TFMS treatment, the molecular weight changes of rrhGM-CSF and yrhGM-CSF were determined by SDSPAGE. rrhGM-CSF has a molecular weight of $24-28 \mathrm{kDa}$, similar to the $1 \mathrm{~N}$-type of human lymphoblastoid Namalwa cell-derived rhGM-CSF (Fig. 2). ${ }^{13,14)}$ After TFMS treatment, its molecular weight was shifted to $15 \mathrm{kDa},{ }^{14)}$ showing that 9-13 kDa of glycans had been removed (Fig. 2). yrhGMCSF has a molecular weight of $16-18 \mathrm{kDa}$ and is known to be $O$-glycosylated. ${ }^{43)}$ Its molecular weight was shifted to $14.5 \mathrm{kDa}$, the same as nonglycosylated hGM-CSF, after TFMS treatment (Fig. 2). ${ }^{714)}$ Thus the glycans of rrhGMCSF represent $37-47 \%$ of its initial molecular weight and the glycans of yrhGM-CSF represent $10-20 \%$ of its initial molecular weight. $N$-Glycosylation sites of yrhGM-CSF were removed by site specific mutation. Therefore, yrhGM-CSF has three potential $O$-glycosylation sites ${ }^{44)}$ whereas rrhGMCSF has two potential $N$-glycosylation sites and three potential $O$-glycosylation sites. ${ }^{9)}$ Glycoproteins containing $N$-gly- cosylation site has a molecular weight greater than the expected value due to the remaining innermost $N$-acetylglucosamine after the TFMS treatment. ${ }^{29)}$ The $15 \mathrm{kDa}$ molecular weight of the deglycosylated rrhGM-CSF was larger than expected value, suggesting that the innermost $N$-acetylglucosamine of $\mathrm{N}$-glycan was not removed by the TFMS treatment. $^{29)}$

Clearance Rate of rhGM-CSF in the Rat We determined the circulating serum protein levels of rrhGM-CSF, yrhGM-CSF, deglycosylated rrhGM-CSF and deglycosylated yrhGM-CSF by sandwich ELISA following intravenous administration to rats. Serum protein levels were measured from 0.25 to $48 \mathrm{~h}$ post injection.

Although yrhGM-CSF had the highest serum protein level up to $4 \mathrm{~h}$, it was cleared rapidly after that and was undetectable $10 \mathrm{~h}$ post injection (Fig. 3). Although the level of rrhGM-CSF was lower than that of yrhGM-CSF up to $4 \mathrm{~h}$, it was cleared more slowly after $10 \mathrm{~h}$ and was $180 \mathrm{pg} / \mathrm{ml}$ at $24 \mathrm{~h}$ and $30 \mathrm{pg} / \mathrm{ml}$ at $48 \mathrm{~h}$ (Fig. 3). The deglycosylated forms of

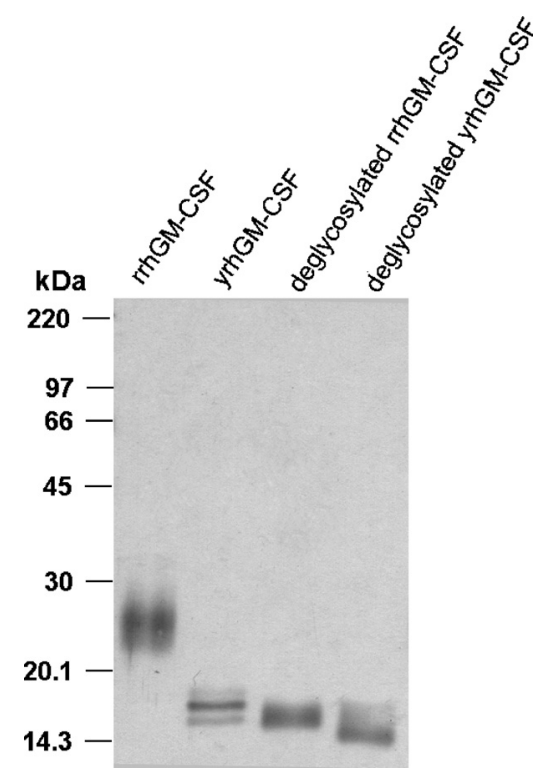

Fig. 2. SDS-PAGE Analysis of Glycosylated rhGM-CSF and Deglycosylated rhGM-CSF

rrhGM-CSF and yrhGM-CSF were deglycosylated by TFMS. Changes of molecular weight were identified by SDS-PAGE. Lane 1, rrhGM-CSF; lane 2, yrhGM-CSF; lane 3, deglycosylated rrhGM-CSF; lane 4, deglycosylated yrhGM-CSF.

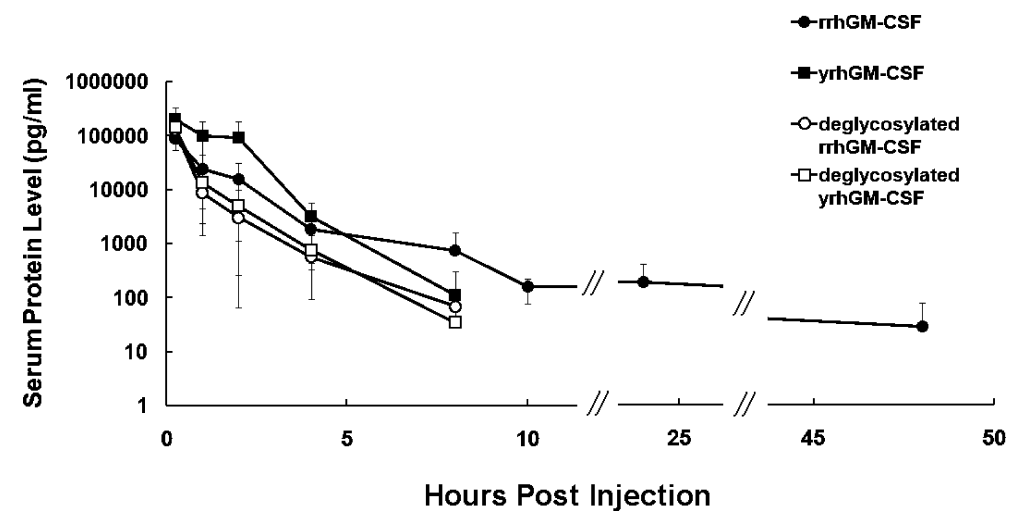

Fig. 3. Circulating Plasma Levels of rhGM-CSF

rhGM-CSF was administrated to 6-week-old male SD rats. rhGM-CSF levels in serum samples were measured by sandwich ELISAs. Data are means \pm S.D. of three rats per group. Data are representative of three independent experiments in which similar results were obtained. 
rrhGM-CSF and yrhGM-CSF were undetectable $10 \mathrm{~h}$ post injection. Therefore, the two deglycosylated rhGM-CSFs have similar clearance rates (Fig. 3). Because rrhGM-CSF persisted for longer than deglycosylated rrhGM-CSF, clearance rate clearly depends on the presence or absence of glycans. However, although yrhGM-CSF had higher serum protein levels than deglycosylated yrhGM-CSF, it was also undetectable $10 \mathrm{~h}$ post injection (Fig. 3). These results indicate that the removed glycans of rrhGM-CSF are very important for in vivo stability and that the different clearance rates of rrhGM-CSF and yrhGM-CSF are due to differences between their individual glycans. Therefore, the glycans of rrhGMCSF is more effective in maintaining in vivo stability than yrhGM-CSF.

In Vitro Bioactivity of rhGM-CSF We measured bioactivities of rhGM-CSFs via dose- and time-dependent manners. Bioactivities of rrhGM-CSF and yrhGM-CSF are similar to each other at 100,500, 1000 and $10000 \mathrm{pg} / \mathrm{ml}$ (Fig. 4A). In addition, $100 \mathrm{pg} / \mathrm{ml}$ of rrhGM-CSF had bioactivity. These findings indicate that the serum protein levels of rrhGM-CSF from $10 \mathrm{~h}$ onwards are valid reflections of their bioactivity (Fig. 3). Proliferation of TF-1 cells was little more increased by rrhGM-CSF than yrhGM-CSF at $24 \mathrm{~h}$ and $48 \mathrm{~h}$, respectively (Fig. 4B). These results indicate that bioactivities of rrhGM-CSF and yrhGM-CSF are similar to each other.

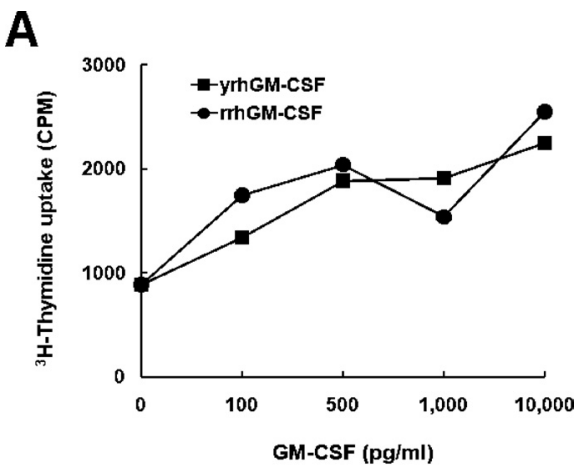

\section{B}

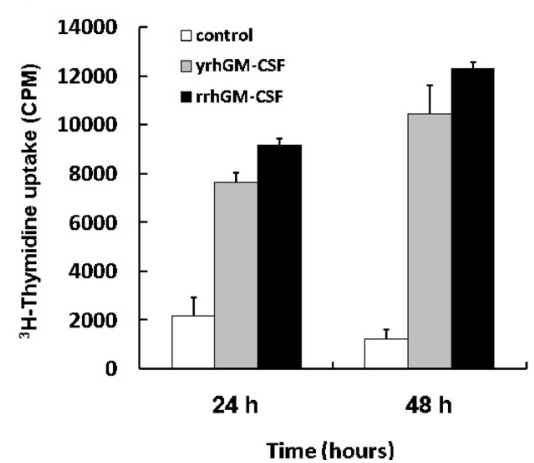

Fig. 4. In Vitro Bioactivities of rhGM-CSF and yrhGM-CSF

The data represent proliferation of TF-1 cells in response to rrhGM-CSF and yrhGM-CSF. Proliferation of TF-1 cells was determined by ${ }^{3} \mathrm{H}$-thymidine uptake as described in Materials and Methods. (A) Dose-dependent effects of rrhGM-CSF and yrhGM-CSF. (B) Time-dependent effects of rrhGM-CSF and yrhGM-CSF. Controls were incubated with culture medium only. Data are means \pm S.D. of duplicate measurements.

\section{DISCUSSION}

hGM-CSF has two potential $N$-glycosylation sites (Asn27, Asn37) and three potential $O$-glycosylation sites (Ser7, Ser9, Thr10).,12,13) It is known that degrees of glycosylation differ according to cell type and that in vivo stability increases with $\mathrm{N}$-glycan content. ${ }^{14)}$ Terminal sialic acids are important for the in vivo stability of glycoproteins. There is no report concerning terminal sialylation of plant cell-derived glycoproteins, although Shah $e a^{23)}$ and Takashima et al. ${ }^{24)}$ reported that plants have the genetic and enzymatic potential for sialylation. Therefore, the lack of terminal sialic acids in plant cell-derived glycoproteins has limited their use as therapeutic agents. ${ }^{20)}$ In this study, we analyzed the terminal sialylation of rrhGM-CSF using lectins. As shown in Fig. 1, mammals like $\alpha 2,3-$ and $\alpha 2,6$ - linked terminal sialic acids were not detected in rrhGM-CSF (Fig. 1A, B). These lectin methods are limited to detect other forms of sialylation. ${ }^{45)}$ Therefore, existence of other form of terminal sialylation can not be excluded.

The glycosylation ratio of rrhGM-CSF was two-fold higher than that of commercial rhGM-CSF (yrhGM-CSF) as shown by experimental deglycosylation of rrhGM-CSF and yrhGM-CSF using TFMS (Fig. 2). Although rrhGM-CSF had lower serum protein levels than yrhGM-CSF up to $4 \mathrm{~h}$ after injection, its level was sustained up to $48 \mathrm{~h}$, whereas yrhGM-CSF was undetectable after $10 \mathrm{~h}$. This result parallels the findings of a pharmacokinetic study by Denzlinger et al. in which rhGM-CSF was administered to humans and serum protein and leukocytes levels were determined ${ }^{46)}$ : heavily glycosylated CHO cell-derived rhGM-CSF gave rise to lower serum protein levels than non-glycosylated Escherichia coliderived rhGM-CSF up to $8 \mathrm{~h}$ but thereafter the opposite was the case. In addition, although the serum protein levels of CHO cell-derived rhGM-CSF were lower than those of Escherichia coli-derived rhGM-CSF up to $10 \mathrm{~h}$, leukocytes numbers were stimulated to the same extent by the two preparation up to $16 \mathrm{~h}$, and from $24 \mathrm{~h}$ onwards the $\mathrm{CHO}$ cellderived rhGM-CSF was more effective in increasing leukocyte numbers. In a report of Okamoto et al., colony formation by human marrow mononuclear cells was no longer increased by rhGM-CSF when its concentration exceeded $100000 \mathrm{pg} / \mathrm{ml} .{ }^{14)}$ This indicates that stimulation of leukocyte multiplication is limited to a certain range of rhGM-CSF concentrations. Therefore, the serum protein level of rhGMCSF from $10 \mathrm{~h}$ is more important for sustaining leukocyte levels than its early level. In our study similarly, although early serum protein levels of rrhGM-CSF were lower than yrhGM-CSF, the level of rrhGM-CSF was better maintained than that of yrhGM-CSF. Hence rrhGM-CSF was more effective than yrhGM-CSF in maintaining leukocyte levels (Fig. 3 ). The stability of rrhGM-CSF in rats depends on the presence of glycans since rhGM-CSF was sustained until $48 \mathrm{~h}$ whereas deglycosylated rrhGM-CSF was undetectable $10 \mathrm{~h}$ post injection. Although yrhGM-CSF was maintained at a higher level than deglycosylated yrhGM-CSF, the latter, like the former, was undetectable $10 \mathrm{~h}$ post injection. Therefore the stability of yrhGM-CSF depends to only a limited extent on the presence of glycans.

Thus, although the glycans on rrhGM-CSF lack terminal sialic acids, rrhGM-CSF is more stable than commercial 
yrhGM-CSF in vivo. Although amino acid sequences of rrhGM-CSF and yrhGM-CSF are different, deglycosylated forms of them have similar clearance rates. Therefore, the glycans on rrhGM-CSF promote in vivo stability more effectively than the glycans on yrhGM-CSF. This property of rrhGM-CSF suggests new possibilities for using transgenic plants in glycoprotein production systems. We are currently performing a detailed study of the in vivo bioactivity and glycosylation profiles of rrhGM-CSF.

Acknowledgements This research was supported by a grant (A18-014) from the Next Generation New Technology Development Project of the Ministry of Commerce, Industry, and Energy of the Republic of Korea.

\section{REFERENCES}

1) Morstyn G., Burgess A. W., Cancer Res., 48, 5624-5637 (1988).

2) Clark S. C., Kamen R., Science, 236, 1229-1237 (1987).

3) Wong G. G., Witek J. S., Temple P. A., Wilkens K. M., Leary A. C., Luxenberg D. P., Jones S. S., Brown E. L., Kay R. M., Orr E. C., Science, 228, 810-815 (1985).

4) Donahue R. E., Wang E. A., Kaufman R. J., Foutch L., Leary A. C., Witek-Giannetti J. S., Metzger M., Hewick R. M., Steinbrink D. R., Shaw G., Cold Spring Harb Symp. Quant. Biol., 51 Pt 1, 685-692 (1986).

5) Moonen P., Mermod J. J., Ernst J. F., Hirschi M., DeLamarter J. F., Proc. Natl. Acad. Sci. U.S.A., 84, 4428-4431 (1987).

6) Libby R. T., Braedt G., Kronheim S. R., March C. J., Urdal D. L., Chiaverotti T. A., Tushinski R. J., Mochizuki D. Y., Hopp T. P., Cosman D., DNA, 6, 221-229 (1987).

7) Burgess A. W., Begley C. G., Johnson G. R., Lopez A. F., Williamson D. J., Mermod J. J., Simpson R. J., Schmitz A., DeLamarter J. F., Blood, 69, 43-51 (1987).

8) Lee J. S., Choi S. J., Kang H. S., Oh W. G., Cho K. H., Kwon T. H., Kim D. H., Jang Y. S., Yang M. S., Mol. Cells, 7, 783-787 (1997)

9) Shin Y. J., Hong S. Y., Kwon T. H., Jang Y. S., Yang M. S., Biotechnol. Bioeng., 82, 778-783 (2003).

10) Kwon T. H., Kim Y. S., Lee J. H., Yang M. S., Biotechnol. Lett., 25, $1571-1574$ (2003).

11) Wang M. L., Goldstein C., Su W., Moore P. H., Albert H. H., Transgenic Res., 14, 167-178 (2005).

12) Kaushansky K., O’Hara P. J., Hart C. E., Forstrom J. W., Hagen F. S., Biochemistry, 26, $4861-4867$ (1987).

13) Forno G., Bollati Fogolin M., Oggero M., Kratje R., Etcheverrigaray M., Conradt H. S., Nimtz M., Eur. J. Biochem., 271, $907-919$ (2004).

14) Okamoto M., Nakai M., Nakayama C., Yanagi H., Matsui H., Noguchi H., Namiki M., Sakai J., Kadota K., Fukui M., Arch. Biochem. Biophys., 286, 562-568 (1991).

15) Dorr R. T., Clin. Ther., 15, 19-29; discussion 18 (1993).

16) Bakker H., Bardor M., Molthoff J. W., Gomord V., Elbers I., Stevens L. H., Jordi W., Lommen A., Faye L., Lerouge P., Bosch D., Proc. Natl.
Acad. Sci. U.S.A., 98, 2899-2904 (2001).

17) Palacpac N. Q., Yoshida S., Sakai H., Kimura Y., Fujiyama K., Yoshida T., Seki T., Proc. Natl. Acad. Sci. U.S.A., 96, $4692-4697$ (1999).

18) Giddings G., Curr. Opin. Biotechnol., 12, 450-454 (2001).

19) Twyman R. M., Stoger E., Schillberg S., Christou P., Fischer R., Trends Biotechnol., 21, 570-578 (2003).

20) Chen M., Liu X., Wang Z., Song J., Qi Q., Wang P. G., Med. Res. Rev., 25, 343-360 (2005).

21) Kasama K., Utsumi J., Matsuo-Ogawa E., Nagahata T., Kagawa Y., Yamazaki S., Satoh Y., J. Interferon Cytokine Res., 15, 407-415 (1995).

22) Ashwell G., Morell A. G., Adv. Enzymol. Relat. Areas Mol. Biol., 41, 99-128 (1974).

23) Shah M. M., Fujiyama K., Flynn C. R., Joshi L., Nat. Biotechnol., 21, 1470-1471 (2003).

24) Takashima S., Abe T., Yoshida S., Kawahigashi H., Saito T., Tsuji S., Tsujimoto M., J. Biochem. (Tokyo), 139, 279-287 (2006).

25) Ohta Y., Tsukada Y., Sugimori T., J. Biochem. (Tokyo), 106, 1086 1089 (1989).

26) Uchida Y., Tsukada Y., Sugimori T., J. Biochem. (Tokyo), 86, 15731585 (1979).

27) Goodarzi M. T., Turner G. A., Glycoconj. J., 14, 493-496 (1997).

28) Patel T., Bruce J., Merry A., Bigge C., Wormald M., Jaques A., Parekh R., Biochemistry, 32, 679-693 (1993).

29) Edge A. S., Biochem. J., 376, 339-350 (2003).

30) Kim H. J., Biol. Pharm. Bull., 30, 1913-1917 (2007).

31) Laemmli U. K., Nature (London), 227, 680 - 685 (1970).

32) Doherty D. H., Rosendahl M. S., Smith D. J., Hughes J. M., Chlipala E. A., Cox G. N., Bioconj. Chem., 16, 1291-1298 (2005).

33) Dunham S. P., Bruce J., Gene, 332, 97-106 (2004).

34) Watson E., Bhide A., van Halbeek H., Glycobiology, 4, 227-237 (1994).

35) Hokke C. H., Bergwerff A. A., Van Dedem G. W., Kamerling J. P., Vliegenthart J. F., Eur. J. Biochem., 228, 981-1008 (1995).

36) Nagano M., Stubiger G., Marchetti M., Gmeiner G., Allmaier G., Reichel C., Electrophoresis, 26, 1633-1645 (2005).

37) Rohrer J. S.,Avdalovic N., Protein Expr. Purif., 7, 39- 44 (1996)

38) Kohli R., Chadha N., Muralidhar K., FEBS Lett., 242, 139-143 (1988).

39) Yun H. Y., Keutmann H. T., Eipper B. A., J. Biol. Chem., 269, 10946-10955 (1994).

40) Jost C. J., Funderburgh J. L., Mann M., Hassell J. R., Conrad G. W., J. Biol. Chem., 266, 13336-13341 (1991).

41) Jarchow J., Fritz J., Anselmetti D., Calabro A., Hascall V. C., Gerosa D., Burger M. M., Fernandez-Busquets X., J. Struct. Biol., 132, 95105 (2000).

42) Lind J. L., Bacic A., Clarke A. E., Anderson M. A., Plant J., 6, 491502 (1994).

43) Ernst J. F., Mermod J. J., Richman L. H., Eur. J. Biochem., 203, 663667 (1992).

44) Gillis S., Urdal D. L., Clevenger W., Klinke R., Sassenfeld H., Price V., Cosman D., Behring Inst. Mitt., 1988, 1-7 (1988).

45) Varki A., FASEB J., 11, 248-255 (1997).

46) Denzlinger C., Tetzloff W., Gerhartz H. H., Pokorny R., Sagebiel S., Haberl C., Wilmanns W., Blood, 81, 2007-2013 (1993). 